\title{
Local Chemistry of Complex Nanostructures and Their Interfaces in Block Copolymer-Templated Nanocomposite Electrodes for Rechargeable Lithium Batteries
}

\author{
V. J. Leppert*, E.C. Nelson**, S. C. Mui***, E. A. Olivetti***, and A. M. Mayes*** \\ *Department of Chemical Engineering and Materials Science, University of California, One Shields \\ Ave., Davis, California, 95616-5294 \\ **National Center for Electron Microscopy, MS 72-150, Berkeley, CA 94720 \\ ***Department of Materials Science and Engineering, Massachusetts Institute of Technology, 13- \\ 5025, 77 Massachusetts Ave., Cambridge, MA 02139
}

Lithium - polymer electrolyte batteries are of great current interest for portable electric power applications, including electric vehicles, due to their light weight and high projected energy densities [1]. Novel electrode materials in these systems incorporate nanoparticles of tin, silver, gold, transition metal oxides such as $\mathrm{LiCoO}_{2}$, iron phosphates, and carbon nanotubes. In appropriate configurations, the nanoparticles allow for fast insertion and extraction of lithium from these materials, and hence high current densities, due to large area to volume ratios [2]. Recently, Mayes and coworkers demonstrated that cycle life can be dramatically extended through the use of a block copolymer electrolyte matrix for the templated assembly of electrodes comprising gold nanoparticles and carbon nanotubes [3]. Analytical characterization at high spatial resolution in the electron microscope using electron energy-loss spectroscopy (EELS) and energy-dispersive X-ray spectroscopy (EDXS) allows the characterization of the local chemistry of the nanostructures and their interfaces in these complex nanocomposites [4,5]. In the case of lithium polymer batteries, elucidation of local chemistry of these materials is expected to lead to a new understanding of the origin of their current and capacity limitations, allowing for the optimization of the electrode configuration.

In this study, the spatial distribution of the respective blocks of the poly(methyl)methacrylate-bpoly(oxyethylene) $)_{9}$ methacrylate, PMMA-b-POEM, block copolymer was determined, as well as their relationship to the single wall carbon nanotubes (SWNT) and gold nanoparticle anodes that are self-assembled in the polymer templating medium. The poly(oxyethylene) ${ }_{9}$ methacrylate, the ionconducting block here, is found to form a bicontinuous gyroid structure that serves to transport the lithium through the battery, while the SWNTs serve as the electron conducting medium.

Microanalysis confirms the presence of metallic gold nanoparticles, formed in-situ in the POEM block from the reduction of $\mathrm{LiAuCl}_{4}$, and serving as an anode material here. The chemistry of the nanostructures and their interfaces are probed at different points in the charging and discharging cycle; and compared to samples consisting of the SWNTs only (Figure 1).

The block copolymer-templated nanocomposite electrodes studied here were prepared by microtoming and subsequently mounted on copper mesh grids, while the SWNT samples were prepared by crushing and dispersal onto copper mesh grids. Data for this study was obtained on a Philips CM-200 TEM operated at $120 \mathrm{kV}$, with an energy resolution of $1.36 \mathrm{eV}$ for Mn K-alpha radiation, and $1 \mathrm{eV}$ EELS energy resolution. Samples were cryogenically cooled during analysis. 
References

[1] D.R. Sadoway and A.M. Mayes, MRS Bulletin 27 (2002) 590.

[2] C.R. Sides, N. Li, C.J. Patrissi, B. Scrosati and C.R. Martin, MRS Bulletin 27 (2002) 604.

[3] S.C. Mui, P.E. Trapa, B. Huang, P.P. Soo, M. L. Lozow, T.C. Wang, R.E. Cohen, A.N.

Mansour, S. Mukerjee, A.M. Mayes and D.R. Sadoway, Journal of the Electrochemical Society 149 (2002) A1610.

[4] P.H. Redlich, D.L. Carroll, P.M. Ajayan, Current Opinion in Solid State and Materials Science 4 (1999) 325.

[5] V.J. Leppert, A.K. Murali, S.H. Risbud, M. Stender, P.P. Power, C. Nelson, P. Banerjee, and A.M. Mayes, Philosophical Magazine B 82 (2002) 1047.

[6] This work was supported by the NSF ADVANCE Fellows Program - Grant \#0137922; the Office of Naval Research under contacts N00014-99-1-0561, N00014-99-1-0565, and N00014-00WX-2-0198; and the NSF MRSEC Program DMR 98-089431. The authors also acknowledge R.E. Smalley's lab at Rice University for supplying SWNT samples under ONR contract N00014-98-10893.

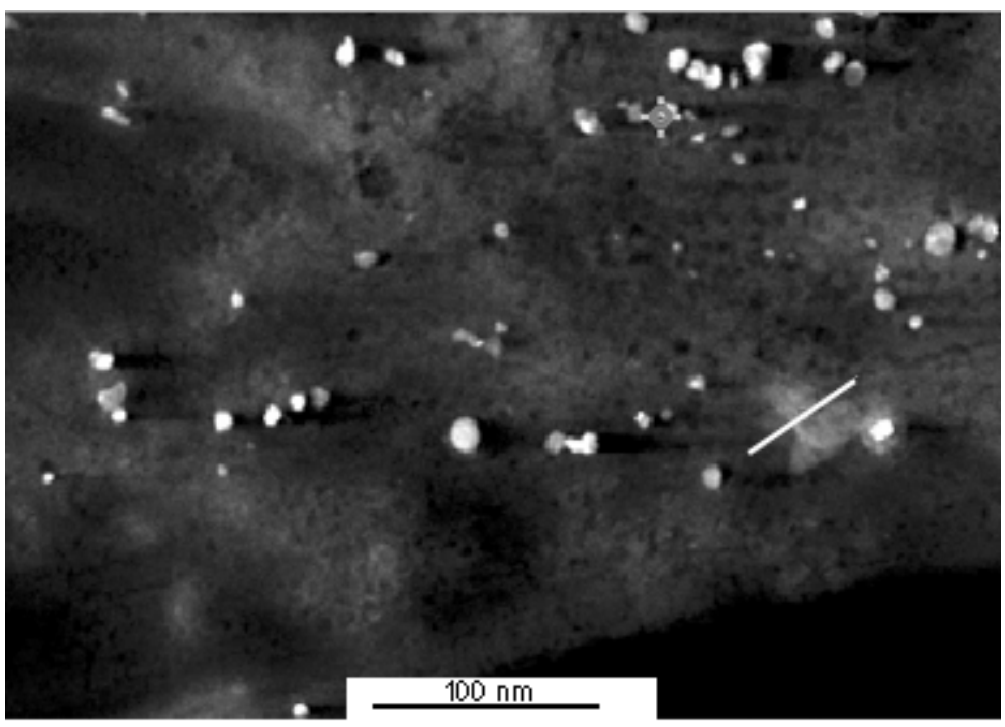

FIG 1. Micrograph of block copolymer lithium battery with SWNT bundle (indicated by line). The larger particles are the $\mathrm{Co} / \mathrm{Ni}$ catalyst particles from the SWNT synthesis. Gold nanoparticles with 1-2 nm diameter that are synthesized in-situ in the POEM block of the copolymer are also visible and confirmed by EDS. 